\title{
Prognostic significance of vascular endothelial growth factor and circulating endothelial cells for early and late outcomes of allogeneic hematopoietic stem cell transplantation
}

Ivan S. Moiseev, Sergej V. Lapin, Elena A. Surkova, Margarita Y. Lerner, Elena V. Babenko, Alexandra A. Sipol, Vladimir N. Vavilov, Boris V. Afanasyev

Correspondence: Ivan S. Moiseev, PhD, Raisa Gorbacheva Memorial Institute of Children Oncology, Hematology and Transplantation, The First St.Petersburg State I. Pavlov Medical University, L. Tolstoy St 6-8, 197022, St. Petersburg, Russia

Phone: +7-812-233-29-25 (office);

+7-921-796-1951 (mobile)

E-mail: moisiv@mail.ru

\section{Summary}

A number of studies evaluated predictive value of endothelial damage markers on outcomes of allogeneic hematopoietic stem cell transplantation (alloHSCT), but they were rarely measured in combination and with long follow up. In this study we evaluated predictive value of vascular endothelial growth factor (VEGF) and circulating endothelial cells (CEC) on early and late non-relapse mortality (NRM). Given the known negative prognostic impact of VEGF expression in hematologic malignancies undergoing chemotherapy, the second goal was to access impact of VEGF level on relapse incidence after alloHSCT. Level of VEGF was analyzed in 91 consecutive patients before the start of conditioning, on day 0 and on the day of engraftment. CEC were measured in 55 consecutive patients from the same study group at the same time points. Both VEGF and CEC were predicted early, but not late NRM. High level of VEGF on day 0 in multivariate analysis was associated with increased 1-year NRM (55\% vs 22\%, HR 3.15, 95\%CI 1.34-7.40, p=0.009), while high level of CEC before conditioning was associat- ed with increased 1-year NRM in univariate (69\% vs $20 \%$, $\mathrm{p}=0.001)$, but not multivariate analysis (95\%CI 0.84-5.76, $\mathrm{p}=0.102$ ). High VEGF A level before conditioning was associated with increased 1-year relapse rate ( $55 \%$ vs $22 \%$, $\mathrm{p}=0.001$, HR 3.15, 95\%CI 1.34-7.40), but had no impact on late relapses and 3-year overall survival (50\% vs $42 \%$ respectively, $\mathrm{p}=0.60$ ), as a large proportion of patients were successfully salvaged after relapse. CEC were not a significant prognostic factor for relapse $(p=0.09)$. No correlation was found between VEGF and CEC at any time point $(\mathrm{p}>0.05)$, indicating that they may represent different aspects of endothelial dysfunction. In conclusion, VEGF and CEC are valuable biomarkers to predict early NRM, and VEGF is also a predictive factor of early relapse after alloHSCT.

\section{Keywords}

hematopoietic stem cell transplantation, endothelial damage, endothelial growth factor, circulating endothelial cells, blood flow, survival, risk factors. 


\section{Introduction}

Allogeneic hematopoietic stem cell transplantation (alloHSCT) is an effective treatment for a number of hematologic malignancies and non-malignant disorders, but it is associated with significant non-relapse mortality (NRM). Recently, a number of studies emphasized the role of endothelial dysfunction in such life-threatening complications of alloHSCT as graft-versus-host disease (GVHD) and veno-occlussive disease (VOD). The concept of endothelial injury in alloHSCT is not new, in 1996 Testa $S$ et al., reported association between level of thrombomodulin, an endothelial damage marker, and GVHD, VOD and septicemia [30]. Since then the role of endothelium in acute alloHSCT complications were predicted to be more complex. On the one hand, neovascularization of bone marrow, skin and gastrointestinal tract is presiding event to acute GVHD, and it is associated with increased level of vascular endothelial growth factor (VEGF) $[13,17,21]$. On the other hand, endothelium is damaged by conditioning and calcineurin inhibitors, which leads to increased incidence of microvascular complications, infections and non-relapse mortality [8]. Also it was proposed that not the overwhelming T-cell alloreactive cytotoxicity, but severe endothelial dysfunction is responsible for steroid-refractory form of acute GVHD [14]. These multiple roles of endothelium in complications of alloHSCT make endothelial damage markers an attractive tool to predict prognosis.

Furthermore, neovasculogenesis and its key regulator VEGF were found to play an important role in pathogenesis of hematologic malignancies. VEGF A expression was associated with altered morphology and increased vascularization of the bone marrow in myeloproliferative disorders $[2,10]$. Also it was demonstrated that increased level of VEGF A is associated with worse outcome of chemotherapy in patients with acute myeloid leukemia [1], chronic myeloid leukemia [34], acute lymphoblastic leukemia [5], myelodysplastic syndrome [33] and different types of lymphoproliferation [6, 11, 25]. Apart from VEGF, level of circulating endothelial cells (CEC) was also found to predict prognosis in patients treated with chemotherapy for acute myeloid leukemia [35]. The significance of this negative prognostic factor was not established in recipients of alloHSCT.

A number of endothelial damage was studied in alloHSCT patients. The principal groups are adhesion molecules (ICAM, VCAM etc.), VEGF, CEC, von Willebrand factor, thrombomodulin and anti-coagulants, which are rapidly depleted in cases of extensive endothelial injury (protein $\mathrm{C}$, antithrombin III etc) [8]. Among all these markers for the present study we selected VEGF A and CECs, because they are increased both in situation of endothelial injury and tumor-associated neovasculogenesis, and thus, may have a prognostic significance for both early complications after alloHSCT and relapse of the underlying hematologic malignancy.

Previously, we have reported a predictive value of VEGF A for early non-relapse mortality and relapses after alloHSCT [20] and a diagnostic significance of CEC level for VOD [19].

In the present study we evaluated predictive significance of VEGF and CECs for short- and long-term outcomes of
alloHSCT and association between these two endothelial damage markers.

\section{Patients and methods Patients}

The study was based on the blood samples and hospital records of 91 consecutive adult patients with hematologic malignancies undergoing HSCT in R.M. Gorbacheva Memorial Institute of Children Hematology and Transplantation. Samples for the study were collected prospectively between 2010 and 2012. The study was approved by the Ethical Committee of I.P. Pavlov State Medical University and informed consent was received from all patients for blood collection.

$73 \%$ of patients had acute leukemia, $10 \%$ chronic myeloid leukemia, $8 \%$ myelodysplastic syndrome and $9 \%$ other hematologic malignancies. Median age was 38 years (range 18-60), median performance score and modified EBMT risk score $[12,29]$ were 1 (range $0-3$ ) and 4 (range 1-6) respectively. 30\% were grafted from related and $70 \%$ from unrelated donor. $24 \%$ received myeloablative conditioning, and $76 \%$, underwent a reduced intensity conditioning. Myeloablative conditioning included $16 \mathrm{mg} / \mathrm{kg}$ oral busulfan and $120 \mathrm{mg} / \mathrm{kg}$ cyclophosphamide. Reduced-intensity conditioning was based fludarabine $180 \mathrm{mg} / \mathrm{m}^{2}$ and $8 \mathrm{mg} / \mathrm{kg}$ busulfan or $140 \mathrm{mg} / \mathrm{m}^{2}$ melphalan in case of previous busulfan-based conditioning or anamnesis of neurological disorders. Acute GVHD prophylaxis consisted of calcineurin inhibitor (tacrolimus in $81 \%$ of patients) and short-course methotrexate. Antilymphocyte globulin (Atgam, Pfizer, NY, USA) $60 \mathrm{mg} / \mathrm{kg}$ was used for unrelated grafts. All but two patients received single allogeneic graft. Ten patients had previous autologous transplantation. Detailed patient characteristics are given in table 1 . VOD prophylaxis with fixed-dose heparin was performed in all patients.

The staging and grading of acute GVHD were performed using the modified Glucksberg consensus criteria [24] and occurred at the time of initiation of treatment. VOD was diagnosed clinically according to the modified Seattle criteria extended until day +30 after HSCT, which required the presence of at least two of the following three clinical findings: jaundice with bilirubin $>34 \mu \mathrm{mol} / \mathrm{l}$, painful hepatomegaly and fluid retention $>5 \%$ of the body weight. VOD was classified as severe in presence of multi-organ failure.

\section{Laboratory methods}

Venous blood was collected using EDTA anticoagulant from the central venous catheter before the start of conditioning, on the day of SCT (day 0) before graft transfusion, on the day of engraftment and additionally on the day of VOD diagnosis.

For VEGF A analysis, the blood samples were centrifuged for 15 minutes at $1000 \mathrm{~g}$ and the plasma aliquots were stored in polypropylene tubes at $-80^{\circ} \mathrm{C}$ until the day of the assay. Measurement of VEGF A concentrations in plasma samples was performed by enzyme-linked immunosorbent assays (ELISA) using commercially available kits (eBioscience, CA, USA) according to the instructions of the manufacturer. The 
detection limit was 7.9 to $1000 \mathrm{pg} / \mathrm{ml}$. Concentrations were determined without knowledge of clinical data.

Measurement of CEC was performed within 2 hours from the blood collection. Peripheral blood samples were processed by lysing buffer (BD Pharm Lyse, BD Biosciences). Cell-surface triple staining was performed with fluorochrome-labeled monoclonal anti-human mouse antibodies incubated for 20 minutes at room temperature. The following antibody combination was used: CD31-FITC, CD146PE, and CD45-PerCP (Becton Dickinson, San Jose, CA,USA). Data acquisition and analysis were performed by using BD FACSAria II flow cytometer and BD FACSDiva software (Becton Dickinson). For CECs measurement at least 1 million events was acquired. To exclude platelets, dead cells, and microparticles, the FSC/SSC plot was used. CECs were identified as CD45-negative CD31-bright positive CD146-bright positive (CD45-/CD31bright/ CD146bright) [16].

\section{Statistical analysis}

Experimental and clinical data were analyzed using SPSS Version 17.0. Chi-square and Wilcoxon criteria were used for univariate non-parametric analysis. Kruskal-Wallis test was used to determine the effect of pre-transplant therapy on the level of endothelial damage markers before conditioning. Kaplan-Meier survival analysis and Log Rank test were used for univariate survival, transplant-related mortality and cumulative incidence of relapse comparisons. Multivariate analysis was performed using Cox regression. Multivariate models were built with the use of stepwise forward selection, using a $\mathrm{p}$-value $\leq 0.01$ to include variables in the model. Proportional-hazards assumption was tested for each variable individually, all variables met this assumption. Separate models were created for assessment of CEC predictive value, since they were measured only in 55 consecutive patients. Cut-off levels of VEGF for survival analysis were determined based on ROC curves. Results of VEGF measurement at dif- ferent time points were selected for Kaplan-Meier analysis and Cox regression if ROC area under the curve (AUC) for this time point was $\geq 0.5$, and the time point with the highest value was selected.

Laboratory samples were collected within 30 days after transplantation and then patients were followed-up for survival and relapse. Median follow-up was 895 days (range 135-1364). NRM was defined as the cumulative incidence of death from the date of transplant not related to the relapse and its subsequent treatment. Even-free survival (EFS) was defined as the time from the date of transplantation to the documented event (relapse or death).

\section{Results}

\section{Clinical outcomes}

Engraftment was achieved in $90 \%$ of patients, $10 \%$ had either primary graft failure and/or progressive disease. Threeyear overall survival (OS), EFS and NRM in the whole group were $44 \%, 35 \%$ and $34 \%$ respectively. Three -year cumulative incidence of relapse was $31 \%$. VOD was diagnosed in $14 \%$ $(n=13)$ of patients, severe VOD- in $10 \%$. Median day of VOD diagnosis was 13 (range 6-21). Acute GVHD grade 1-4 was diagnosed in $54.4 \%$ of patients, grade $3-4$ GVHD in $20.9 \%$.

\section{Selection of the optimal time points and cut-offs}

The ROC curve analysis was performed to determine the time points of alloHSCT with highest predictive value for NRM and RR. The analysis separated early events (within 1 year after alloHSCT) and late events (after 1 year). The analysis for late relapses was not performed since there was only single such event in the study group. The results for VEGF A and CEC are presented in table 1.

\begin{tabular}{|l|l|l|l|}
\hline Time point & $\begin{array}{l}\text { Before } \\
\text { conditioning }\end{array}$ & Day 0 & $\begin{array}{l}\text { Upon } \\
\text { engraftment }\end{array}$ \\
\hline VEGF A & \multicolumn{4}{|l|}{} \\
\hline 1-year relapse incidence & 0.62 & 0.53 & 0.50 \\
\hline 1-year non-relapse mortality & 0.46 & 0.67 & 0.57 \\
\hline Relapse incidence after one year & n.a & n.a & n.a \\
\hline Non-relapse mortality after one year & 0.40 & 0.36 & 0.46 \\
\hline Circulating endothelial cells & \multicolumn{3}{l}{} \\
\hline 1-year relapse incidence & 0.55 & 0.62 & 0.62 \\
\hline 1-year non-relapse mortality & 0.66 & 0.50 & 0.54 \\
\hline Relapse incidence after one year & n.a & n.a & n.a \\
\hline Non-relapse mortality after one year & 0.54 & 0.35 & 0.00 \\
\hline
\end{tabular}

n.a.= not applicable

Table 1. ROC areas under the curves for predictive value of VEGF $A$ and CECs 
VEGF A before conditioning was found to have the highest predictive value for 1 -year relapse $(\mathrm{AUC}=0.62)$. The ROC determined cut-off for relapse was $37 \mathrm{pg} / \mathrm{ml}$. An additional analysis was performed to exclude the effect of pre-transplant chemotherapy on the level of VEGF A before conditioning. Neither time from diagnosis to HSCT $(p=0.83)$, number of previous chemotherapy courses $(p=0.12)$, number of high-dose chemotherapy courses $(p=0.82)$, previous HSCT $(p=0.11)$ or time from the last chemotherapy course $(\mathrm{p}=0.28)$ significantly effected the level of VEGF A.

Early NRM was best predicted by VEGF A level after conditioning (AUC=0.67). The determined cut-off value was the limit of quantification $(<7.8 \mathrm{pg} / \mathrm{ml})$. On the contrary, late NRM was not predicted by VEGF A concentrations with AUC $<0.5$ at all time points.

For CECs, early relapse was best predicted by level after conditioning ( $\mathrm{AUC}=0.62$ ) with a cut-off 87 cells $/ \mathrm{ml}$. Level of CECs before conditioning was both predictive for 1-year (AUC=0.66) and late NRM (AUC=0.54) with a cut-off value of 38 cells $/ \mathrm{ml}$. As with VEGF time from diagnosis to HSCT $(\mathrm{p}=0.34)$, number of previous chemotherapy courses $(\mathrm{p}=0.40)$, number of high-dose chemotherapy courses $(p=0.58)$ or time from the last chemotherapy course $(p=0.25)$ were not significantly effecting the pre-transplant level of CECs.

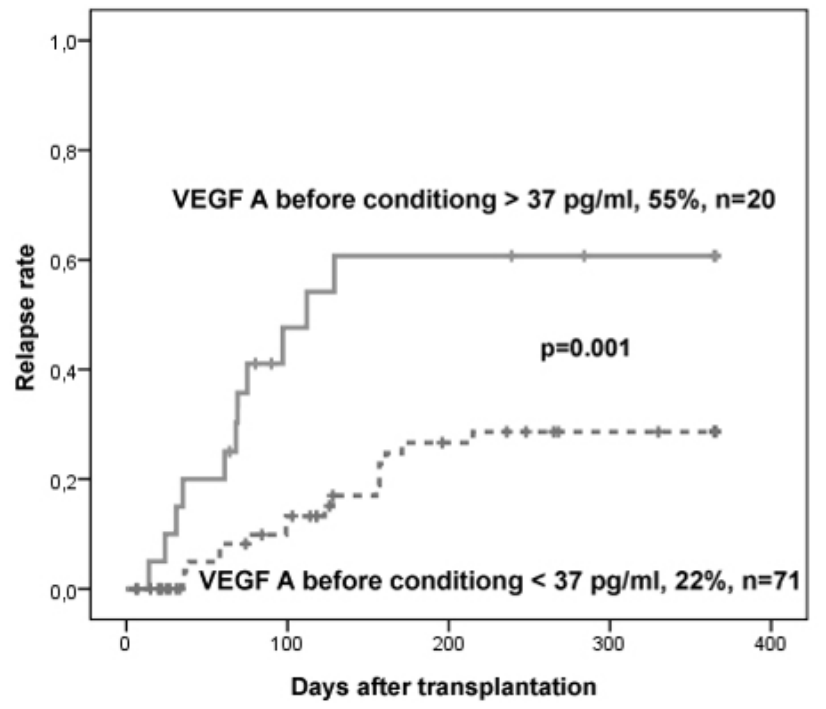

As based on these results, we selected pre-transplant VEGF A level and day 0 CEC level to be tested in Kaplan-Meier survival analysis of relapse incidence, and day 0 VEGF along with pre-transplant CEC levels for NRM analysis.

\section{Predictive value of VEGF for relapse and non-relapse mortality}

Patients with pre-conditioning level of VEGF A $>37 \mathrm{pg} / \mathrm{ml}$ had significantly higher 1-year relapse incidence (55\% vs $22 \%, \mathrm{p}=0.001$, HR 3.15, 95\%CI 1.34-7.40, figure 1). Also patients VEGF A after conditioning $<7.8 \mathrm{pg} / \mathrm{ml}$ demonstrated a trend to lower non-relapse mortality ( $17 \%$ vs $35 \%, \mathrm{p}=0.10$, Fig. 1). In a multivariate analysis VEGF $A>37 \mathrm{pg} / \mathrm{ml}$ was a significant factor associated with relapse (HR 3.15, 95\%CI 1.34-7.40, $\mathrm{p}=0.009$, table 2). Moreover, when corrected for confounding factors in multivariate regression VEGF A after conditioning $<7.8 \mathrm{pg} / \mathrm{ml}$ was a significant predictor of NRM (HR 0.15, 95\%CI 0.03-0.69, p=0.015, table 2). Surprisingly, 3 -year overall survival (OS) was not different in patients with high and low VEGF concentrations ( $50 \%$ vs $42 \%$ respectively, $\mathrm{p}=0.60$ ), as majority of patients in high VEGF group responded to salvage therapy after relapse.

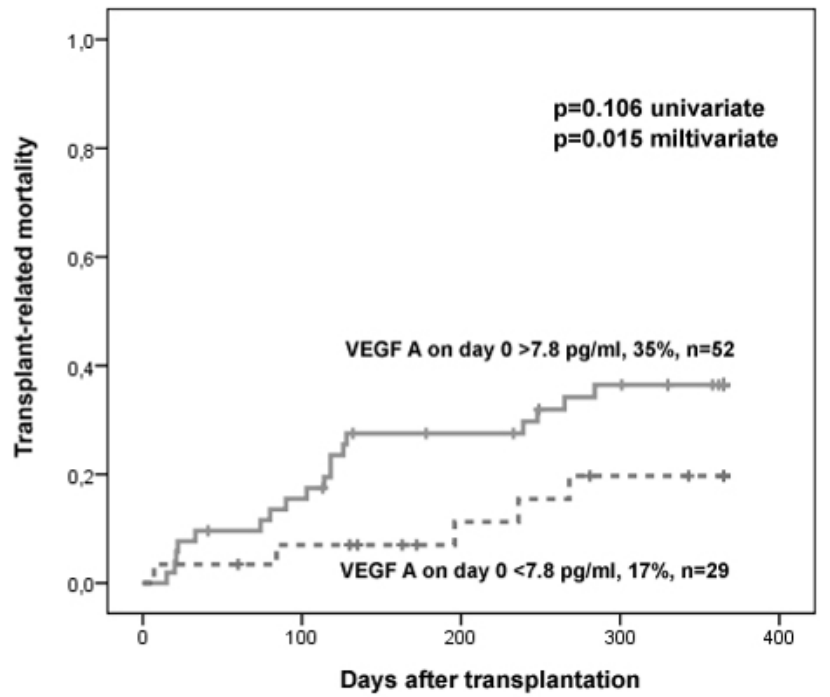

Figure 1. 1-year relapse incidence and non-relapse mortality according to VEGF level 


\begin{tabular}{|l|l|l|l|}
\hline Factor & Multivariate, p-value & HR & HR 95\% Cl \\
\hline $\begin{array}{l}|l| \\
\text { 1-year cumulative relapse rate }\end{array}$ & 0.232 & & \\
\hline Unrelated vs related donor & 0.002 & 4.05 & $1.68-9.79$ \\
\hline Active disease at the time of HSCT & 0.461 & & \\
\hline MAC vs RIC conditioning & 0.002 & 0.26 & $0.11-0.61$ \\
\hline Acute GVHD, grade 1-4 & 0.003 & 0.19 & $0.06-0.55$ \\
\hline Chronic GVHD & 0.009 & 3.15 & $1.34-7.40$ \\
\hline VEGF A before conditioning > 37 pg/mI & & & \\
\hline 1-year non-relapse mortality & 0.027 & 4.67 & $1.19-18.30$ \\
\hline Active disease at the time of HSCT & 0.70 & & \\
\hline Age & 0.022 & 6.64 & $1.32-33.40$ \\
\hline Unrelated vs related donor & 0.32 & & \\
\hline MAC vs RIC conditioning & 0.024 & 4.87 & $1.24-19.14$ \\
\hline Acute GVHD, grade 3-4 & 0.015 & 0.15 & $0.03-0.69$ \\
\hline VEGF A on day $0<7.8 \mathrm{pg} / \mathrm{ml}$ & & \\
\hline
\end{tabular}

Table 2. Multivariate analysis of relapse incidence and non-relapse mortality (whole group)

\section{Predictive value of CECs for relapse and non-relapse mortality}

Univariate analysis with previously determined cut-off value showed patients with CEC level after conditioning $>87$ cells/ $\mathrm{ml}$ had higher relapse incidence ( $60 \%$ vs $21 \%, \mathrm{p}<0.001)$, but in multivariate analysis it was not a significant factor $(\mathrm{p}=0.09$, table 3). So CECs was not an independent predictive marker of relapse, but rather demonstration the increased endothelial damage in patients with high-risk disease. This was confirmed by the fact that level $>87$ cells $/ \mathrm{ml}$ was significantly more often observed in patients with active disease at the time of the alloHSCT ( $80 \%$ vs $20 \%, \mathrm{p}=0.032$ ).
Univariate analysis of NRM confirmed that patients with pre-conditioning level of CECs $>71$ had increased risk of transplant-related death during the first year (69\% vs $20 \%$, $\mathrm{p}=0.001$ ), while the incidence of late NRM was not significantly different ( $0 \%$ vs $5 \%, \mathrm{p}=0.70$ ). Nevertheless, in multivariate analysis the differences in 1-year NRM were also not significant (95\%CI 0.84-5.76, p=0.102, table 3). Regarding results of multivariate analysis of CEC as the predictive marker, the statistical power of the models was relatively low due to small number of patients with CEC measured, so with a larger group of patients, the CECs as the covariate might reach the significant level.

\begin{tabular}{|l|l|l|l|}
\hline Factor & Multivariate, p-value & HR & HR 95\% Cl \\
\hline \multicolumn{2}{|l|}{ 1-year cumulative relapse rate } & & \\
\hline Active disease at the time of HSCT & 0.82 & & \\
\hline MAC vs RIC conditioning & 0.148 & 0.24 & $0.07-0.91$ \\
\hline Acute GVHD, grade 1-4 & 0.035 & 0.08 & $0.01-0.66$ \\
\hline Chronic GVHD & 0.019 & & \\
\hline CEC after conditioning>87 cells/mI & 0.086 & & \\
\hline 1-year non-relapse mortality & \multicolumn{2}{|l|}{} \\
\hline Active disease at the time of HSCT & 0.050 & 3.05 & $1.00-9.31$ \\
\hline Acute GVHD, grade 3-4 & 0.040 & 2.98 & $1.05-8.45$ \\
\hline CEC before conditioning>71 cells/ml & 0.102 & & \\
\hline
\end{tabular}

Table 2. Multivariate analysis of relapse incidence and non-relapse mortality (only patients with measured level of CECs) 


\section{Association between level of VEGF $A$ and CECS}

The interesting result of the analysis was that there was no correlation between pre-transplant level of CECs and VEGF $(\mathrm{p}=0.81)$, as well as for after conditioning $(\mathrm{p}=0.74)$ and engraftment levels $(\mathrm{p}=0.59)$. But when the cut-off levels of CEC $(>71$ cells $/ \mathrm{ml})$ and VEGF $(<7.8 \mathrm{pg} / \mathrm{ml})$ that predicted NRM were tested, in patients with high CEC level pre-conditioning VEGF was significantly more frequently elevated on day 0 ( $83 \%$ vs $51 \%, \mathrm{p}=0.036)$.

\section{Discussion}

In this study we confirmed the prognostic significance of endothelial damage markers for non-relapse mortality. As expected, these markers allow to predict only early, but not late mortality. This is due to the fact that late NRM is mostly associated with chronic GVHD or infections related to impaired immune system [26]. The factors that influence development of chronic GVHD and immune reconstitution are mostly immunologic and are associated with patient-donor major or minor HLA-antigen disparity and trigger these events in the late post-transplant period $[3,24]$. On the contrary, in an early post-transplant period endothelial dysfunction plays a role in a number of acute complications. In our study due to small number of patients it was impossible to establish relationship between endothelial damage and all of the complications, but as we previously reported elevation of VEGF and CEC levels was closely related to development of VOD $[19,20]$. Likewise, a number of studies demonstrated association between endothelial damage and acute GVHD [14, $15,18]$, and it was demonstrated in non-transplant patients that endothelial dysfunction results in higher incidence of infectious complications and worse prognosis in patients with sepsis [22, 31].

In this study we found out that VEGF is predictive for NRM after conditioning, while CEC - when measured before conditioning. So different endothelial damage markers may represent different aspects of endothelial damage and are elevated variable time after a damage occurs. This was confirmed in our study by absence of correlation between VEGF and CEC, and only more sophisticated methods revealed association between these two biomarkers. So VEGF A is secreted a large variety of cells, including endothelial and blood mononuclears. It is eliminated via cleavage with serum proteases and binding to either soluble or cellular VEGF receptors. But also a large proportion of VEGF is bound to extracellular matrix (ECM) heparin sulfate proteoglycans. In response to endothelial and tissue damage ECM-bound VEGF is cleaved from proteoglycan complex and released into circulation to facilitate reparation. What is important is that activity of serum proteases in terms of VEGF elimination is insufficient to decrease its level in situations of its extensive release from ECM. But its levels in plasma return to normal values very rapidly, within hours, if there are no continuous stimuli [9, $28,32]$. Hence, VEGF A level in plasma represent the acute phase of endothelial damage, that is why the time-point post-conditioning is of highest predictive value. CEC levels, on the other hand, represent chronic endothelial damage and are present in circulation for a longer period of time than VEGF. That is why it is a valuable tool to predict complications in diabetes and cardiovascular disorders [3, 7]. Marginally significant predictive value of CEC in multivariate analysis may not indicate that this is less valuable prognostic marker, but may be a result of a smaller number of patients in whom it as measured. In summary, different endothelial damage markers complement each other and in further clinical research should be used in combination.

Another aspect of our study was a dual role of VEGF. Besides its role as endothelial damage biomarker, it is also a disease risk factor in hematologic malignancies [1, 5, 6, 11, 33, 34]. We have demonstrated that it is also a factor of adverse disease prognosis in HSCT, but it is important that there were no differences in OS respective to pre-transplant VEGF level. Successful salvage post-transplant chemotherapy and donor lymphocyte infusions, at least in some patients from the high-VEGF A group, demonstrates a potential utility of graft-versus-leukemia effect to overcome this factor of resistance. Allogeneic HSCT is considered a treatment of choice for high-risk disease in patients with acute leukemias, myelodysplastic syndrome, chronic myeloid leukemia and lymphomas $[23,36]$. As standard therapy outcome for these malignancies was reported to be negatively influenced by VEGF A overexpression, further studies are required to determine whether patients with high level of this factor should be candidates for HSCT. Due to very low incidence of late relapses in the study group we failed to demonstrate if there was any influence of VEGF expression on late disease recurrence, so larger studies are required to test this, but given very high incidence of early relapses in our study in the high VEGF group, early post-transplant therapy should be considered for these patients.

Recently, a number of clinical scoring systems were validated to predict risk of alloHSCT, like EBMT risk score [12,29] or Seattle PAM score [27]. The results of this study show that the endothelial damage markers are of distinct predictive value, irrelevant of clinical parameters. Therefore, further studies are required to evaluate if they could be incorporated into the risk assessment models. In conclusion, peripheral CECs and plasma VEGF levels represent a useful prognostic tool to predict the risk of alloHSCT and disease relapse.

\section{Conflict of interests}

No conflict interest is reported. 


\section{References}

1. Aguayo A, Estey E, Kantarjian $\mathrm{H}$ et al. Cellular vascular endothelial growth factor is a predictor of outcome in patients with acute myeloid leukemia. Blood 1999; 94(11): 3717-21.

2. Aguayo A, Kantarjian H, Manshouri T et al. Angiogenesis in acute and chronic leukemias and myelodysplastic syndromes. Blood 2000; 96(6): 2240-5.

3. Allen JL, Fore MS, Wooten J et al. B cells from patients with chronic GVHD are activated and primed for survival via BAFF-mediated pathways. Blood 2012; 120(12): 2529-36.

4. Asicioglu E, Gogas Yavuz D, Koc M, Ozben B, Yazici D, Deyneli O, Akalin S. Circulating endothelial cells are elevated in patients with type 1 diabetes mellitus. Eur J Endocrinol 2010; 162(4): 711-7.

5. Avramis IA, Panosyan EH, Dorey F, Holcenberg JS, Avramis VI. Children's Oncology Group. Correlation between high vascular endothelial growth factor-A serum levels and treatment outcome in patients with standard-risk acute lymphoblastic leukemia: a report from Children's Oncology Group Study CCG-1962. Clin Cancer Res 2006; 12(23): 6978-84.

6. Ben Arush MW, Ben Barak A, Maurice S, Livne E. Serum VEGF as a significant marker of treatment response in Hodgkin lymphoma. Pediatr Hematol Oncol 2007; 24(2): 111-5.

7. Boos CJ, Balakrishnan B, Blann AD, Lip GY. The relationship of circulating endothelial cells to plasma indices of endothelial damage/dysfunction and apoptosis in acute coronary syndromes: implications for prognosis. J Thromb Haemost 2008; 6(11): 1841-50.

8. Carreras E, Diaz-Ricart M. The role of the endothelium in the short-term complications of hematopoietic SCT. Bone Marrow Transplant 2011; 46(12): 1495-502.

9. Chen TT, Luque A, Lee S, Anderson SM, Segura T, Iruela-Arispe ML. Anchorage of VEGF to the extracellular matrix conveys differential signaling responses to endothelial cells. J Cell Biol 2010; 188(4): 595-609.

10. de Bont ES, Rosati S, Jacobs S, Kamps WA, Vellenga E. Increased bone marrow vascularization in patients with acute myeloid leukaemia: a possible role for vascular endothelial growth factor. Br J Haematol 2001; 113(2) :296-304.

11. Hatjiharissi E, Terpos E, Papaioannou M et al. The combination of intermediate doses of thalidomide and dexamethasone reduces bone marrow micro-vessel density but not serum levels of angiogenic cytokines in patients with refractory/relapsed multiple myeloma. Hematol Oncol 2004; 22(4): 159-68.

12. Hemmati PG, Terwey TH, le Coutre P et al. A modified EBMT risk score predicts the outcome of patients with acute myeloid leukemia receiving allogeneic stem cell transplants. Eur J Haematol. 2011; 86(4): 305-16.

13. Leonhardt F, Grundmann S, Behe M et al. Inflammatory neovascularization during graft-versus-host disease is reg- ulated by av integrin and miR-100. Blood. 2013; 121(17): 3307-18.

14. Luft T, Dietrich S, Falk C et al. Steroid-refractory GVHD: T-cell attack within a vulnerable endothelial system. Blood 2011; 118(6): 1685-92.

15. Lunn RA, Sumar N, Bansal AS, Treleaven J. Cytokine profiles in stem cell transplantation: possible use as a predictor of graft-versus-host disease. Hematology 2005; 10(2): 107-14.

16. Mancuso P, Antoniotti P, Quarna J et al. Validation of a standardized method for enumerating circulating endothelial cells and progenitors: flow cytometry and molecular and ultrastructural analyses. Clin Cancer Res 2009;15(1): 267-73.

17. Medinger M, Tichelli A, Bucher C et al. GVHD after allogeneic haematopoietic SCT for AML: angiogenesis, vascular endothelial growth factor and VEGF receptor expression in the BM. Bone Marrow Transplant. 2013; 48(5): 715-21.

18. Min CK, Kim SY, Lee MJ et al. Vascular endothelial growth factor (VEGF) is associated with reduced severity of acute graft-versus-host disease and nonrelapse mortality after allogeneic stem cell transplantation. Bone Marrow Transplant 2006; 38(2): 149-56.

19. Moiseev IS, Babenko EV, Sipol AA et al. Measurement of circulating endothelial cells to support the diagnosis of veno-occlusive disease after hematopoietic stem cell transplantation. Int J Lab Hematol. 2014; 36(4): e27-9.

20. Moiseev IS, Lapin SV, Surkova EA et al. Level of vascular endothelial growth factor predicts both relapse and nonrelapse mortality after allogeneic hematopoietic stem cell transplantation. Biol Blood Marrow Transplant. 2013; 19(12): 1677-82.

21. Norton J, Sloane JP, al-Saffar N et al. Vessel associated adhesion molecules in normal skin and acute graft-versus-host disease. J Clin Pathol. 1991; 44(7): 586-91.

22. Page AV, Liles WC. Biomarkers of endothelial activation/ dysfunction in infectious diseases. Virulence 2013; 4(6): 507-16.

23. Phillips GL. Allogeneic hematopoietic stem cell transplantation (HSCT) for high-risk acute myeloid leukemia (AML)/myelodysplastic syndrome (MDS): how can we improve outcomes in the near future? Leuk Res. 2012; 36(12): 1490-5.

24. Przepiorka D, Weisdorf D, Martin P et al. Consensus conference on acute GVHD grading. Bone Marrow Transplant 1995; 15(6): 825-828.

25. Riihijärvi S, Nurmi $H$, Holte $H$ et al. High serum vascular endothelial growth factor level is an adverse prognostic factor for high-risk diffuse large B-cell lymphoma patients treated with dose-dense chemoimmunotherapy. Eur J Haematol 2012; 89(5): 395-402.

26. Schechter T, Pole JD, Darmawikarta D et al. Late mortality after hematopoietic SCT for a childhood malignancy. Bone Marrow Transplant. 2013; 48(10): 1291-5. 
27. Sorror ML, Storer BE, Maloney DG et al. Outcomes after allogeneic hematopoietic cell transplantation with nonmyeloablative or myeloablative conditioning regimens for treatment of lymphoma and chronic lymphocytic leukemia. Blood. 2008; 111(1): 446-52.

28. Stefanini MO, Wu FT, Mac Gabhann F, Popel AS. A compartment model of VEGF distribution in blood, healthy and diseased tissues. BMC Syst Biol 2008; 2: 77.

29. Terwey TH, Hemmati PG, Martus $P$ et al. A modified EBMT risk score and the hematopoietic cell transplantation-specific comorbidity index for pre-transplant risk assessment in adult acute lymphoblastic leukemia. Haematologica. 2010; 95(5): 810-8.

30. Testa S, Manna A, Porcellini A et al. Increased plasma level of vascular endothelial glycoprotein thrombomodulin as an early indicator of endothelial damage in bone marrow transplantation. Bone Marrow Transplant. 1996; 18(2): 383-8.

31. Vassiliou AG, Mastora Z, Orfanos SE et al. Elevated biomarkers of endothelial dysfunction/activation at ICU admission are associated with sepsis development. Cytokine. 2014; 69(2): 240-7.
32. Vempati P, Mac Gabhann F, Popel AS. Quantifying the proteolytic release of extracellular matrix-sequestered VEGF with a computational model. PLoS One 2010; 5(7): e11860.

33. Verstovsek S, Estey E, Manshouri T et al. Clinical relevance of vascular endothelial growth factor receptors 1 and 2 in acute myeloid leukaemia and myelodysplastic syndrome. Br J Haematol 2002; 118(1): 151-6.

34. Verstovsek S, Kantarjian H, Manshouri T et al. Prognostic significance of cellular vascular endothelial growth factor expression in chronic phase chronic myeloid leukemia. Blood 2002; 99(6): 2265-7.

35. Wierzbowska A, Robak T, Krawczynska A et al. Kinetics and apoptotic profile of circulating endothelial cells as prognostic factors for induction treatment failure in newly diagnosed acute myeloid leukemia patients. Ann Hematol. 2008; 87(2): 97-106.

36. Zikos P, Van Lint MT, Lamparelli T et al. Allogeneic hemopoietic stem cell transplantation for patients with high risk acute lymphoblastic leukemia: favorable impact of chronic graft-versus-host disease on survival and relapse. Haematologica. 1998; 83(10): 896-903. 